\title{
Evaluation of Kidney Injury Molecule-1 (KIM-1) and Neutrophil Gelatinase-Associated Lipocalin (NGAL) Urinary Levels for Detecting Kidney Dysfunction in Patients With Nasopharyngeal Cancer Treated With Cisplatin-Based Treatment
}

\author{
Marliana Sri Rejeki ${ }^{1}$, Wawaimuli Arozal ${ }^{1}$, Rianto Setiabudy ${ }^{1}$, Djumhana \\ Atmakusuma ${ }^{2}$ \\ ${ }^{1}$ Department of Clinical Pharmacology, Faculty of Medicine University of Indonesia, Jakarta, Indonesia \\ ${ }^{2}$ Department of Internal Medicine, Division of Hematology and Medical Oncology, University of Indonesia, Jakarta, \\ Indonesia
}

\section{ARTICLE INFO}

\section{Article history:}

Received : 21 July 2018

Reviewed: 15 August 2018

Accepted : 23 August 2018

\section{Keywords:}

cisplatin, kidney dysfunction, nasopharyngeal cancer, urinary KIM-1, urinary NGAL
* Corresponding author: Marliana Sri Rejeki Department of Internal Medicine, Division of Hematology and Medical Oncology, University of Indonesia, Jakarta, Indonesia email: marliana.sr@gmail.com

\begin{abstract}
A B STRACT
Background: Cisplatin has a potency of causing nephrotoxicity. Serum BUN and creatinine levels have been well-known for detecting kidney dysfunction; while KIM-1 and NGAL urine levels are relatively new measurements. The study was aimed to evaluate urinary KIM-1 and NGAL level to detect kidney dysfunction in patients with advanced stage NPC who received cisplatin-based chemotherapy.
\end{abstract}

Methods: The study was a cohort-prospective study with 3 subject groups, i.e. patients who had never received and who had received $75-100 \mathrm{mg} / \mathrm{m} 2$ cisplatin-based chemotherapy as well as those who had never received $40 \mathrm{mg} / \mathrm{m} 2$ cisplatin-based chemotherapy. The levels of urinary KIM-1, NGAL and serum level of BUN and creatinine were measured before and after receiving cisplatin. Statistical analyses were ANOVA, Pearson, Spearman, Kolmogorov-Smirnov test and SPSS version 22.0.

Result: There was a significant difference of delta BUN level $(p=0.0001)$ and delta urinary NGAL level $(p=0.025)$ before and after treatment in all three groups; while delta KIM-1 level showed no significant difference in all three groups $(p=0.275)$. Cisplatin may cause accumulated nephrotoxicity, which has dose-dependent manner.

Conclusion: Measuring urinary NGAL level can detect an early stage of kidney dysfunction; however, it still cannot replace the role of BUN. Measurement of urinary KIM-1 level cannot detect kidney dysfunction.

\section{INTRODUCTION}

Cisplatin is the main treatment for nasopharyngeal carcinoma. The cancer is rare worldwide, but it has an essential clinical importance as it is commonly misdiagnosed and has a remarkable geographical and racial distribution. Nasopharyngeal carcinoma (NPC) is widely found in Asia, particularly in China and South East Asia, including Indonesia. The global incidence of NPC is $1: 100,000(1,2)$. In Indonesia, the incidence may reach 6.2 per 100,000 citizens annually, or it is assumed that there are 13,000 new cases per year (3). The incidence may also depend on racial and geographical distribution as different areas may affect different histological and etiological appearance (4). The cancer may bring a relatively huge economic burden to health care cost of a country since the cancer is mostly present in male patients at their productive age (1).

Cisplatin has been known as a highly effective chemotherapeutic drug for NPC (6). However, it has also been described as a drug with quite significant side effects and toxicities. One of the most common toxicities of cisplatin is nephrotoxicity which may cause kidney dysfunction (7). Kidney dysfunction due to cisplatin nephrotoxicity is usually started with the development of apoptosis of the proximal tubule cells 
that continually develops into acute kidney injury in the form of acute necrosis. It is assumed that long-term cisplatin treatment may cause the development of cystic tubules. Moreover, Yao et al. (2) have also assumed that the degree of severity of the necrosis is dose-dependent and time-dependent. Yao et al. (2) have also suggested that about $20 \%$ of patients who had high-dose cisplatin treatment are likely to have severe kidney dysfunction. However, no definite study has clearly elaborated the effect on nephrotoxicity due to low-dose cisplatin treatment. Other studies have also demonstrated that nephrotoxicity caused by cisplatin is extremely dosedependent $(2,3)$. Acute kidney failure, either with or without oliguria, may develop in 2-6 days after the patient has been given high-dose cisplatin treatment, while chronic kidney failure may occur due to a set of intravenous cisplatin of at least $20 \mathrm{mg} / \mathrm{m} 2 /$ day for 5 days which is given once in every 3 weeks. The chronic kidney dysfunction may last for over 2 years (3).

Kidney dysfunction caused by the anticancer treatment should be detected early. Usually, kidney damage can be detected by measuring the serum levels of blood urea nitrogen (BUN) and creatinine, which are easily calculated and providing fast results (4). Yao et al. (2) have suggested that the concentration of cisplatin in proximal tubule cells may reach 5 folds of the serum concentration. Therefore, it is expected that measurement using urinary levels of biomarker can provide a more accurate evaluation on the kidney function than using the serum level of creatinine and urea. Some biomarkers may provide a quite promising result to detect kidney dysfunction including the neutrophil gelatinase-associated lipocalin (NGAL) and kidney injury molecule-1 (KIM-1). It is expected that NGAL can be used as a biomarker for detecting the early stage of damage in cortical tubules of the kidney, while KIM-1 may exhibit a relatively good sensitivity to detect proximal tubular damage of the kidney, either the damage is acute, sub-chronic or chronic (10).

Nevertheless, studies on biomarkers using urinary levels of KIM-1 and NGAL to predict the presence of kidney dysfunction in patients with nasopharyngeal patients treated with cisplatin had never been conducted in Indonesia. Therefore, our study was aimed to evaluate the urinary KIM-1 and NGAL levels for detecting kidney dysfunction in patients with NPC who were treated with cisplatin-based chemotherapy.

\section{MATERIAL AND METHOD}

This study is a cohort prospective study conducted in patients with NPC who had been treated with cisplatinbased chemotherapy. The study was conducted in Cipto Mangunkusumo Hospital, Jakarta, Indonesia and data collection was performed after an ethical clearance had been issued (No. 987/UN2.F1/ETIK/2016) by the Ethics
Committee at Faculty of Medicine, University of Indonesia and (No. LB.02.01/X2/10942016) by Cipto Mangunkusumo Hospital. Blood and urinary sampling were performed on patients with NPC who were hospitalized at the A ward in the Department of Internal Medicine, Division of Hematology and Medical Oncology of Cipto Mangunkusumo Hospital. The study was conducted for three months starting from December 2016 to February 2017. Data management was performed at the Laboratory of Pharmacology and 24hour Clinical Pathology Laboratory at Cipto Mangunkusumo Hospital. The technique used for sampling was consecutive sampling.

Patients who became our study subjects were patients with NPC who had cisplatin-based chemotherapy at the dose of $75-100 \mathrm{mg} / \mathrm{m} 2$ and 40 $\mathrm{mg} / \mathrm{m} 2$. The subjects were categorized into 3 groups: (1) NPC patients who had never been exposed to cisplatinbased chemotherapy of $75-100 \mathrm{mg} / \mathrm{m} 2$; (2) NPC patients who had been treated with cisplatin-based chemotherapy at least a cycle of $75-100 \mathrm{mg} / \mathrm{m} 2$ dose; (3) NPC patients who had never been exposed to cisplatin-based chemotherapy of $40 \mathrm{mg} / \mathrm{m} 2$ dose.

Measurements of KIM-1 and NGAL urinary levels, and BUN and creatinine serum levels were performed on day 0 and day 5 (before and after cisplatin treatment) in NPC patients who received cisplatin-based chemotherapy at $75-100 \mathrm{mg} / \mathrm{m} 2$ dose (group 1 and 2) and the measurements were done on day 0 for those who received cisplatin-based chemotherapy at 40 $\mathrm{mg} / \mathrm{m} 2$ dose (group 3). (Figure 1)

The inclusion criteria were: (1) Patients with NPC aged 18-59 years old who had never been exposed to cisplatin-based chemotherapy of adjusted dose to their clinical condition of $75-100 \mathrm{mg} / \mathrm{m} 2$; (2) Patients with NPC aged 18-59 years old who had received cisplatinbased chemotherapy of adjusted dose to their clinical condition of $75-100 \mathrm{mg} / \mathrm{m} 2$ for at least one cycle; (3) Patients with NPC aged 18-59 years old who had never been exposed to cisplatin-based chemotherapy of 40 $\mathrm{mg} / \mathrm{m} 2.5$ The exclusion criteria were patients with severe disease (end-stage chronic kidney failure, heart failure) so that they could not participate and complete the study and patients who were unwilling to participate the study. Eligible patients were recruited as study subjects and underwent measurement of urinary KIM-1 and NGAL as well as serum BUN and creatinine levels. The measurement of urinary KIM-1 and NGAL levels was performed at the Laboratory of Pharmacology, Faculty of Medicine, University of Indonesia. Details of the procedure are provided below, while the measurement of serum BUN and creatinine levels was carried out in the 24-hour Clinical Pathology Laboratory at Cipto Mangunkusumo Hospital, Jakarta 


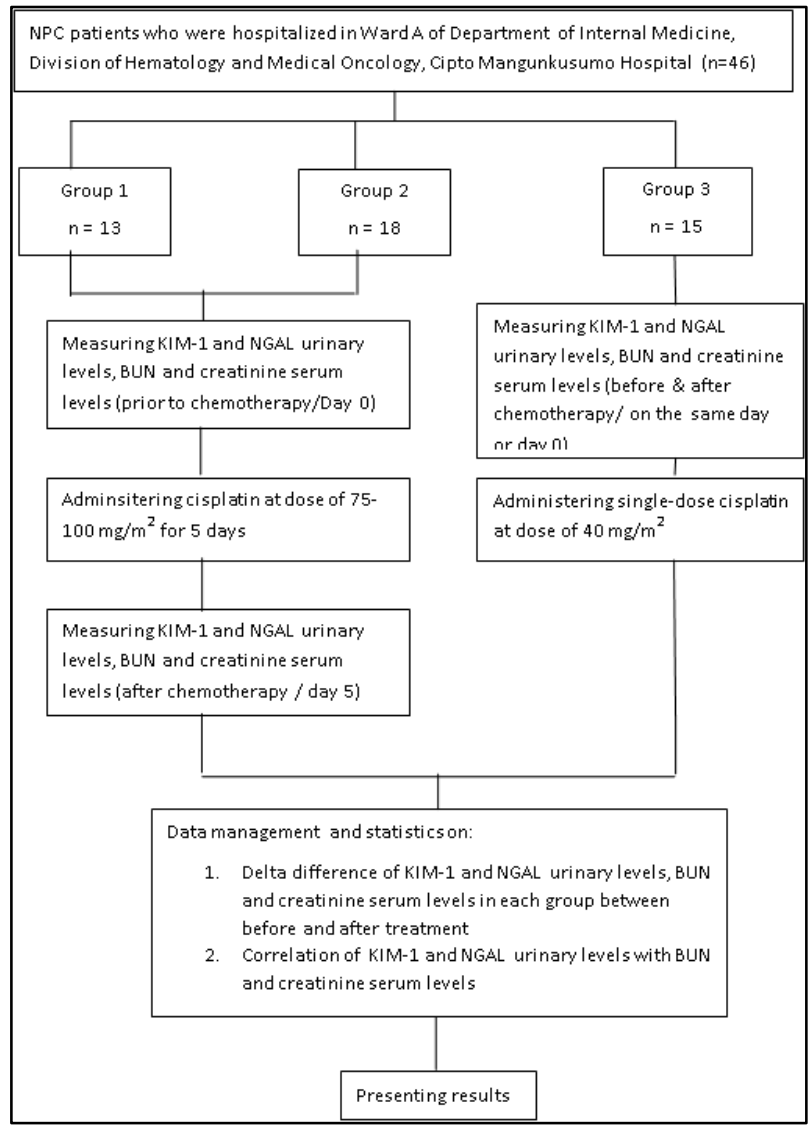

Figure 1. Algorithm of the study flow

\section{Measurement of KIM-1 and NGAL in urine}

The measurement of urinary KIM-1 and NGAL level was carried out using an ELISA kit (Human KIM-1 and Human NGAL, Elabscience, catalogue number: E-ELH0186 and E-EL-H0096) with Sandwich-ELISA method. Urine samples were collected from study subjects to measure urinary KIM-1 and NGAL level. We collected first voided morning urine sample in sterile containers and stored at $-20^{\circ} \mathrm{C}$. All taps of samples were tightened to prevent evaporation and microbial contamination. In the laboratory, urine samples were centrifuged for 20 minutes at $1000 \mathrm{~g}$ at $2-8^{\circ} \mathrm{C}$ and subsequently the supernatants were analysed immediately. Before performing the analysis, standard reagent was prepared and brought to room temperature $\left(18-25^{\circ} \mathrm{C}\right)$ within 15 minutes before use.

Urine samples and standard reagent are added to the appropriate micro ELISA plate wells and combined with specific antibody. Then a biotinylated detection antibody specific for KIM-1/NGAL and AvidinHorseradish Peroxidase (HRP) conjugate is added to each micro plate well and incubated. Free components are washed away using wash buffer. The substrate solution was added to each well. The well containing KIM-1/NGAL biotinylated detection antibody and avidinHRP conjugate appeared in blue colour. The enzymesubstrate reaction was terminated by addition of a sulphuric acid solution and the color turned yellow. The optical density (OD) was measured spectrophotometrically at the wavelength of $450 \mathrm{~nm}+2$ $\mathrm{nm}$. The OD value was proportional to the concentration of KIM-1/ NGAL. The concentrations of KIM-1/ NGAL in the samples were measured by comparing the OD of the samples to the standard curve.

Univariate and bivariate analyses were used in our study. Univariate analysis was performed to identify the correlation between urinary KIM-1 and NGAL levels and BUN and creatinine serum levels in each group using scatter plot diagram. Bivariate analysis was used to measure delta difference of KIM-1 and NGAL urinary level and delta difference of BUN and creatinine serum levels to compare kidney function before and after having cisplatin-based chemotherapy in each group. ANOVA test was used in bivariate analysis when the data distribution was normal, while Kruskal Wallis test was used when the data distribution was abnormal. The $p$ value of $\leq 0.05$ was determined as the acceptable significance level. Data was presented in the form of text, tabels, and box plots using Graphpad Prism Version 5.01. Both univariate and bivariate analyses were performed using SPSS version 22.0. Afterwards, multi comparison analysis was performed using post-hoc Tukey test.

\section{RESULTS}

Total subjects who participated in the study were 46 patients. The number of subjects who had received cisplatin-based chemotherapy of $75-100 \mathrm{mg} / \mathrm{m} 2$ dose was 31 subjects that consisted of 13 subjects of NPC patients in the group that had never been exposed to prior chemotherapy and 18 NPC patients who had received chemotherapy of at least 1 cycle. Our study also included data of 15 subjects who had cisplatinbased chemotherapy with a dose of $40 \mathrm{mg} / \mathrm{m} 2$. All subjects had completed the study and no subject was lost to follow up.

Data on subject characteristics of all groups was comparable as can be seen in table 1.

Evaluation of kidney dysfunction based on the correlation between KIM-1 and NGAL urinary levels and BUN and creatinine serum levels

In general, our study showed that the NGAL and KIM1 urinary level did not have any correlation and had a weak correlation with BUN and creatinine serum levels in almost all groups. However, a strong correlation between creatinine serum level and NGAL urinary level was found in one group, i.e. the group that had never received chemotherapy of $75 \mathrm{mg}-100 \mathrm{mg} / \mathrm{m} 2$. (Figure 2 and Figure 3) 
Table 1. Subject Characteristics

\begin{tabular}{|c|c|c|c|c|c|c|c|}
\hline \multirow{2}{*}{\multicolumn{2}{|c|}{ Variables }} & \multicolumn{2}{|c|}{$\begin{array}{c}\text { Group } 1 \\
n=13\end{array}$} & \multicolumn{2}{|c|}{$\begin{array}{c}\text { Group } 2 \\
n=18\end{array}$} & \multicolumn{2}{|c|}{$\begin{array}{c}\text { Group } 3 \\
n=15\end{array}$} \\
\hline & & Mean \pm SD & Number \% & Mean \pm SD & Number \% & Mean \pm SD & Number \% \\
\hline \multirow[t]{6}{*}{ Characteristics } & Male & & $12(92.31)$ & & $14(77.78)$ & & $10(66.67)$ \\
\hline & Female & & $1(7.69)$ & & $4(22.28)$ & & $5(33.33)$ \\
\hline & Age (years) & $42.62 \pm 12.29$ & & $49.56 \pm 13.82$ & & $42.67 \pm 12.23$ & \\
\hline & Weight(kg) & $53.38 \pm 12.06$ & & $54.47 \pm 12.94$ & & $52.50 \pm 7.91$ & \\
\hline & Height $(\mathrm{cm})$ & $158.15 \pm 16.86$ & & $164.22 \pm 7.89$ & & $162.07 \pm 8.26$ & \\
\hline & Body surface $\left(\mathrm{mm}^{2}\right)$ & $1.47 \pm 0.24$ & & $1.56 \pm 0.20$ & & $1.55 \pm 0.13$ & \\
\hline \multirow[t]{9}{*}{ Ethnics } & Bataknese & & $1(7.69)$ & & $1(5.56)$ & & $1(6.67)$ \\
\hline & Batamian & & $1(7.69)$ & & & & \\
\hline & Bengkulunese & & & & $1(5.56)$ & & \\
\hline & Javanese & & $1076.92)$ & & $12(66.67)$ & & $11(73.33)$ \\
\hline & Chinese descendants & & & & $1(5.56)$ & & $2(13.33)$ \\
\hline & Padangnese & & $1(7.69)$ & & $3(16.67)$ & & $1(6.67)$ \\
\hline & Bogorian & & & & & & \\
\hline & Floresian & & & & & & \\
\hline & Jakartan & & & & & & \\
\hline \multirow[t]{7}{*}{ Occupation } & Civil servants/ & & & & & & \\
\hline & Pension & & $1(7.69)$ & & $1(5.56)$ & & $3(20)$ \\
\hline & Private sector & & & & & & \\
\hline & employee & & $7(53.85)$ & & $6(33.33)$ & & $5(33.33)$ \\
\hline & Housewives & & $1(7.69)$ & & $3(16.67)$ & & $3(20)$ \\
\hline & Entrepreneurs & & $2(15.38)$ & & $6(33.33)$ & & $3(20)$ \\
\hline & Students & & $2(15.38)$ & & $2(11.11)$ & & $1(6.67)$ \\
\hline
\end{tabular}
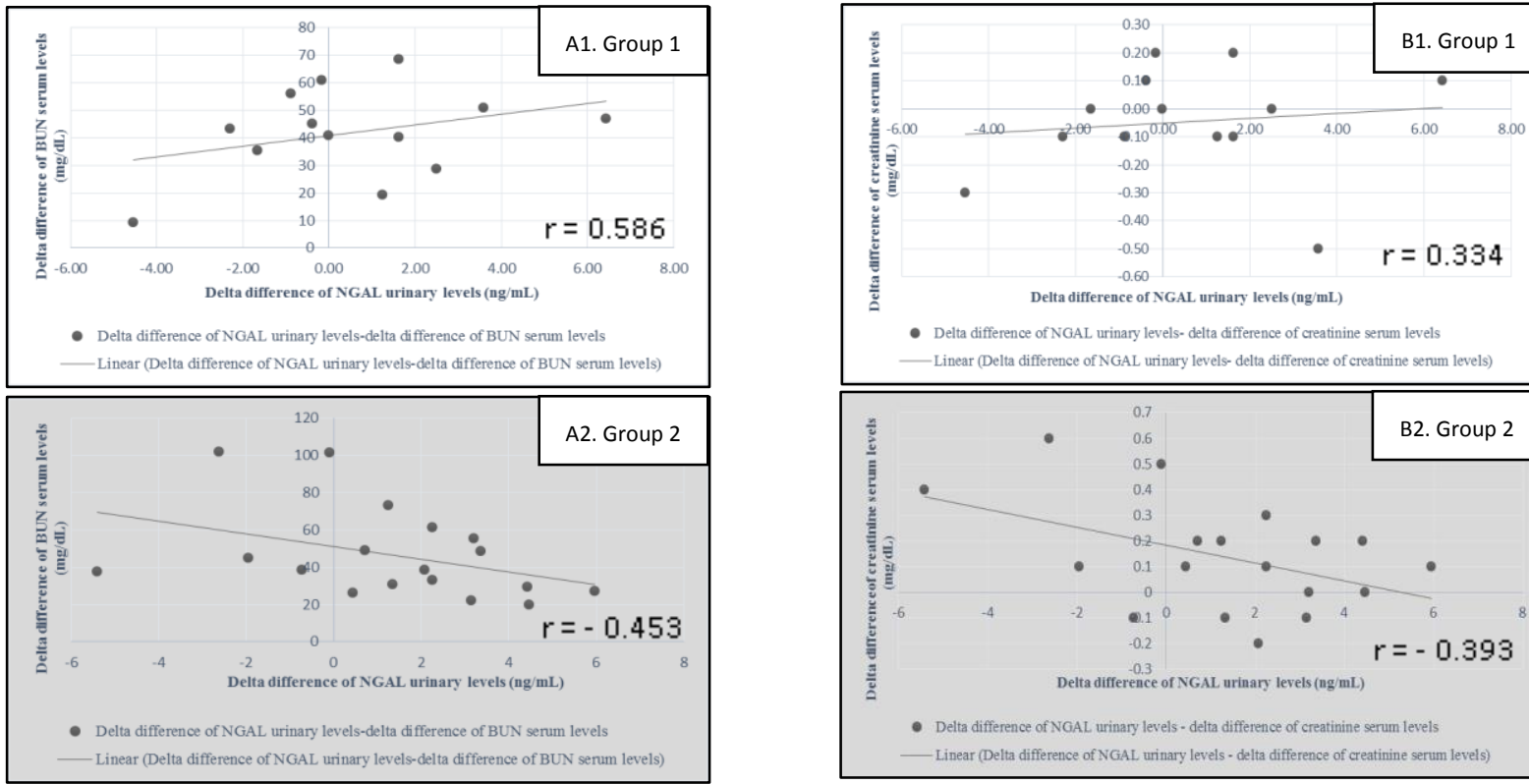

- Linear (Delta difference of NGAL urinary levels- delta difference of creatinine serum levels

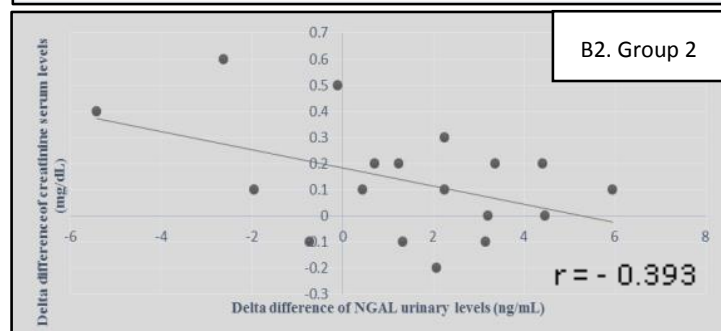

- Delta difference of NGAL urinary levels - detta difference of creatinine serum levels

- Linear (Delta difference of NGAL urinary levels - delts difference of creatinine serum levels)
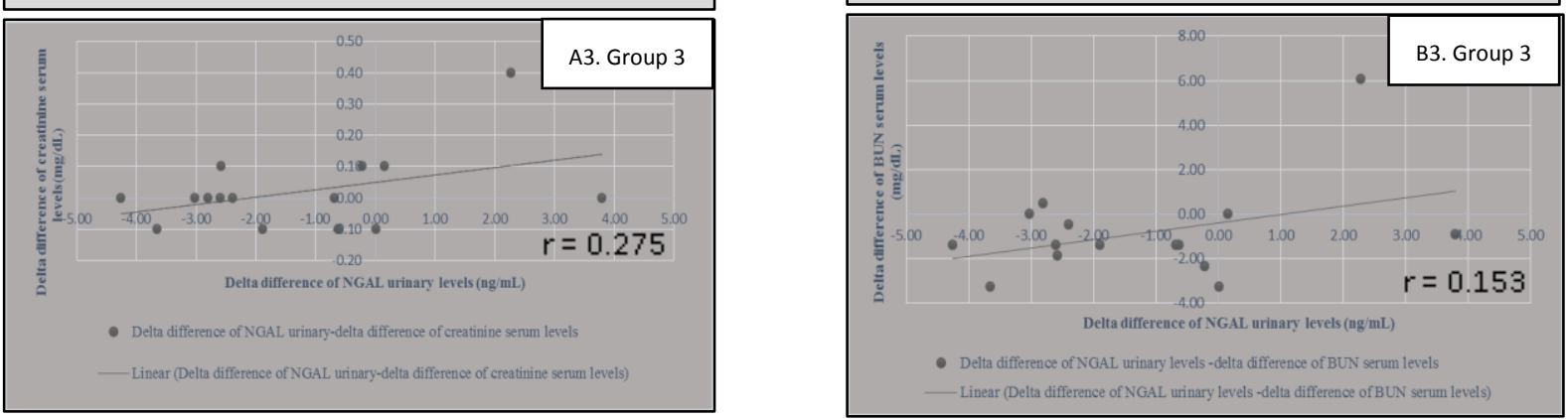

Figure 2. Correlation between delta difference of NGAL urinary levels and delta difference of BUN and creatinine serum levels. (A) Correlation between delta difference of NGAL urinary levels and delta difference of creatinine serum levels in three groups. (B) Correlation between delta difference of NGAL urinary levels and delta difference of BUN serum levels in three groups: Group 1: NPC patients who had never been exposed to cisplatin-based chemotherapy of $75-100 \mathrm{mg} / \mathrm{m}^{2}$ Group 2: NPC patients who had been treated with cisplatin-based chemotherapy at least a cycle of $75-100 \mathrm{mg} / \mathrm{m}^{2}$ dose Group 3: NPC patients who had never been exposed to cisplatin-based chemotherapy of $40 \mathrm{mg} / \mathrm{m}^{2}$ dose. 

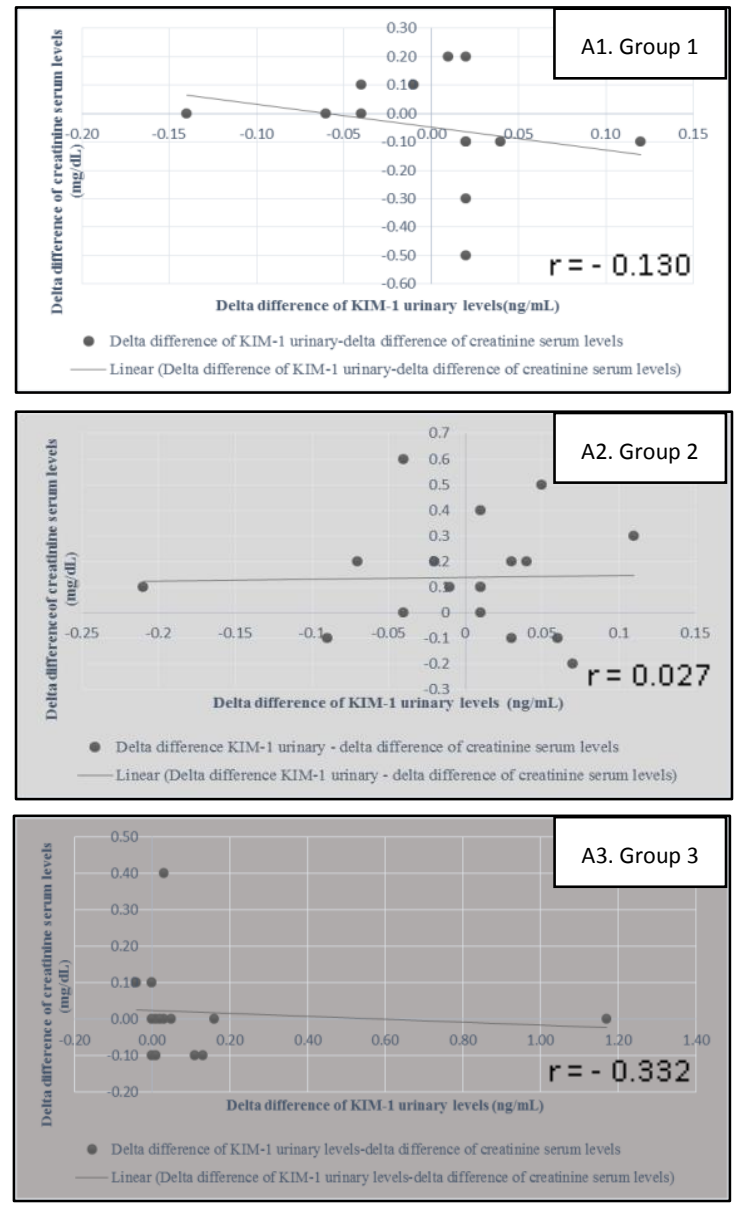

Figure 3. Correlation between delta difference of KIM-1 urinary levels and delta difference of creatinine and BUN serum levels. (A) Correlation between delta difference of KIM-1 urinary levels and delta difference creatinine serum levels in three groups; (B) Correlation between KIM-1 urinary levels and delta difference BUN serum levels in three groups: Group 1: NPC patients who had never been exposed to cisplatin-based chemotherapy of 75-100 mg/m2; Group 2: NPC patients who had been treated with cisplatin-based chemotherapy at least a cycle of $75-100 \mathrm{mg} / \mathrm{m} 2$ dose; Group 3: NPC patients who had never been exposed to cisplatin-based chemotherapy of $40 \mathrm{mg} / \mathrm{m} 2$ dose.

\section{Evaluation of kidney dysfunction based on delta difference of BUN and creatinine serum level and KIM- 1 and NGAL urinary level}

The delta difference of BUN and creatinine serum levels as well as KIM-1 and NGAL urinary levels between before and after chemotherapy treatment can be seen in figure 4. Our study showed that the delta difference of NGAL urinary level was normally distributed and therefore, an ANOVA test was performed. In contrast, the delta difference of KIM-1 urinary level, creatinine and BUN serum levels were not normally distributed, in which the Kruskal Wallis took place.

Data of our study demonstrated that there was a significant delta difference of NGAL urinary level
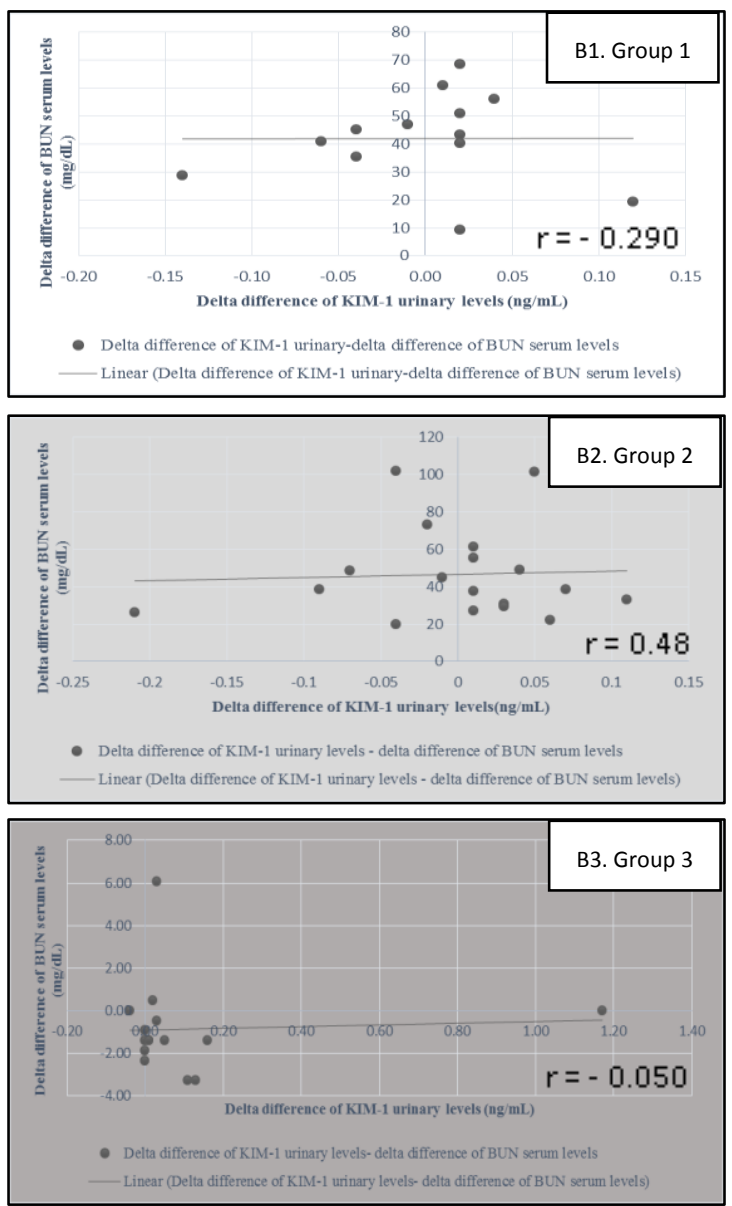

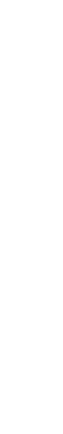



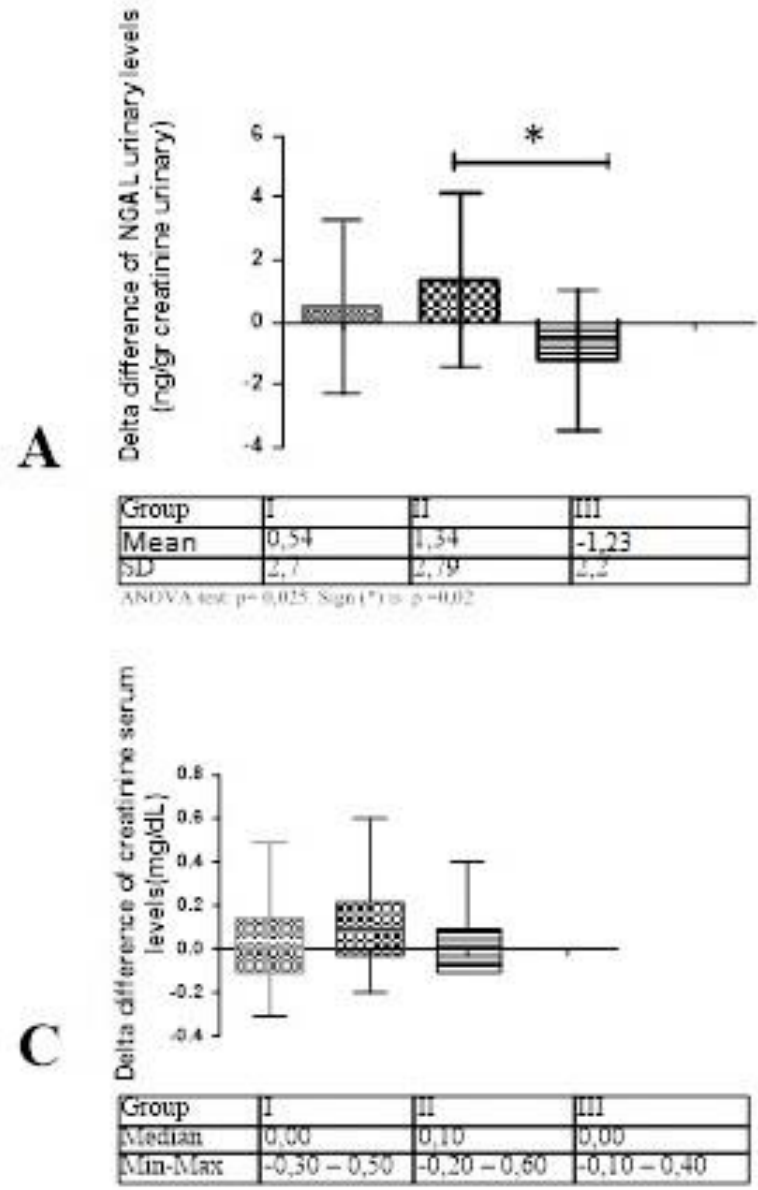
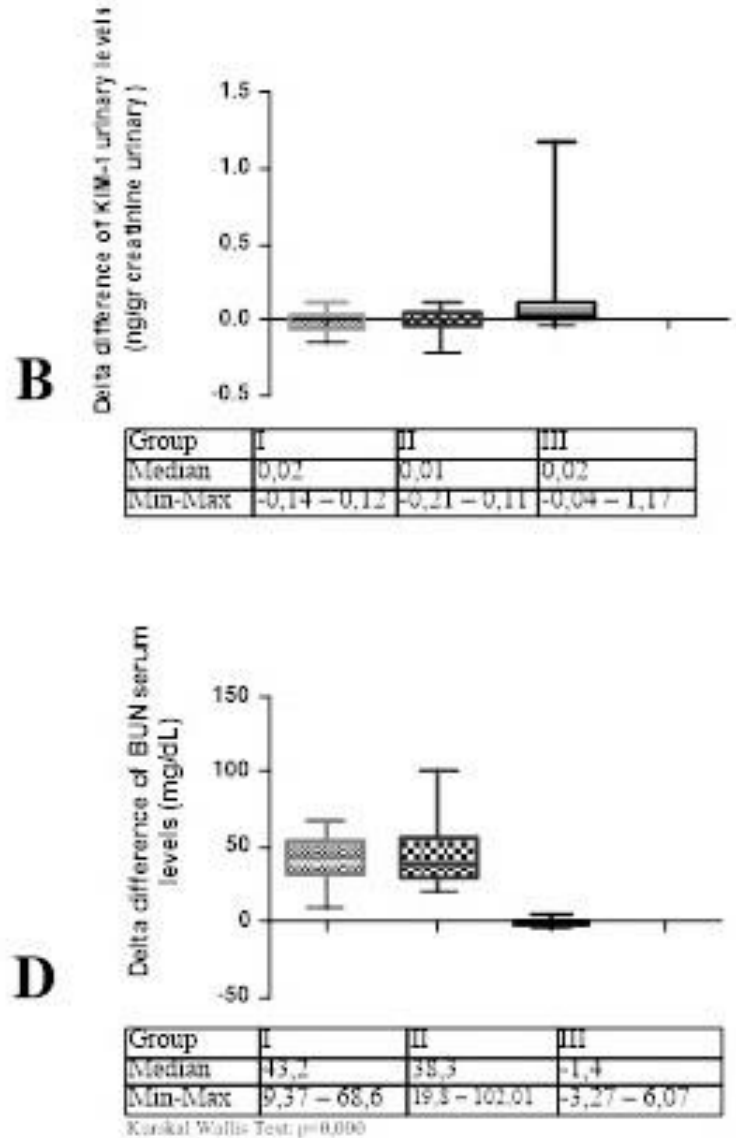

Groups 1: Patients with NPC aged 18-59 years old who had never been exposed to cisplatin-based chemotherapy of adjusted dose to their clinical condition of $75-100 \mathrm{mg} / \mathrm{m}^{2}$

Group 2: Patients with NPC aged 18-59 years old who had received cisplatun-based chemotherapy of adjusted dose to their climical condition of $75-100 \mathrm{mg} / \mathrm{m}^{2}$ for at least one cycle.

\section{Group 3: Patients with NPC aged 18-59 years old who had never been exposed to cisplatin-based} chemotherapy of $40 \mathrm{mg} / \mathrm{m}^{2}$

Figure 4. Delta difference of KIM-1 urinary levels, NGAL urinary levels, BUN and creatinine serum levels between before and after chemotherapy in three groups. (A) Delta of NGAL urinary levels; (B) Delta of KIM-1 urinary levels; (C) Delta of creatinine serum levels; (D) Delta of BUN serum levels

\section{DISCUSSION}

Chemotherapy is the main treatment for advanced stage carcinoma. However, the use of Chemotherapy often becomes limited due to its side effects. Cisplatin is the main chemotherapy for advanced stage NPC. It may cause kidney dysfunction or has a nephrotoxic characteristic. However, data about the effect of cisplatin as NPC chemotherapy is still very limited, particularly the data about kidney dysfunction that may develop.
Kidney dysfunction caused by the anticancer treatment should be detected early. In general, kidney damage can be detected by measuring the serum levels of creatinine and blood urea nitrogen (BUN). Other than BUN and creatinine serum levels, some biomarkers were assumed to be sensitive and reliable to establish the diagnosis of kidney dysfunction including the urinary levels of KIM-1 (kidney injury molecule-1) and NGAL (neutrophil gelatinase-associated lipocalin). Measuring the levels of those biomarkers in the urine has also been assumed that the biomarker can predict earlier acute kidney damage. 
The results of the study showed that cisplatin can cause kidney dysfunction as shown by a significant delta difference of BUN serum level between before and after treatment in three groups $(p=0.0001)$.

Our study also showed that there was a higher delta difference of BUN serum level in subjects receiving cisplatin-based chemotherapy of $75-100 \mathrm{mg} / \mathrm{m} 2$ compared to those receiving at $40 \mathrm{mg} / \mathrm{m} 2$ dose. It indicates that there is a kidney dysfunction due to cisplatin, which is dose dependent and there is an accumulation effect of cisplatin dose on nephrotoxicity. The dose dependent effect of cisplatin in our study is obvious in subjects who had never received cisplatin, but then subsequently received cisplatin at $40 \mathrm{mg} / \mathrm{m} 2$. Those subjects had a lower delta difference of BUN serum level compared to the subjects receiving 75-100 $\mathrm{mg} / \mathrm{m} 2$ cisplatin as they received far less exposure of cisplatin.

Similar results have also been shown by other studies. Espeli et al. (5) have reported that there is a significant reduction of kidney function in patients who had been given cisplatin at the dose of $40 \mathrm{mg} / \mathrm{m} 2$ compared to patients who had been given $100 \mathrm{mg} / \mathrm{m} 2(p=0.04)$. Rades et al. (6) have demonstrated that there is a significant reduced kidney function in patients with $20 \mathrm{mg} / \mathrm{m} 2$ cisplatin compared to those receiving $100 \mathrm{mg} / \mathrm{m} 2$ cisplatin ( $1 \%$ vs. $8 \%$ patients with third degree of kidney dysfunction). Moreover, the accumulation effect of cisplatin in our study has also been showed by a higher delta difference of BUN serum level in subjects who had received several cycles of $75-100 \mathrm{mg} / \mathrm{m} 2$ cisplatin than those who had never received chemotherapy. Cisplatin can be accumulated in the kidney, increases membrane fragility of tubular cells, and causing acute tubular necrosis and tubular dilatation that can be manifested as increased BUN and creatinine serum levels. Arunkumar et al. (7) in their study has demonstrated the nephrotoxicity of cisplatin in cervical cancer, esophageal cancer, head and neck cancer as well as showing that cisplatin can be accumulated in the kidney and the nephrotoxicity occurs in dose dependent manner.

In daily clinical practice, kidney dysfunction is usually detected by measuring BUN and creatinine serum level. In our study, there was a significant delta difference of BUN serum level between before and after treatment in three groups $(p=0.000)$, while the creatinine serum level was not significantly different $(p=0.140)$. It suggests that cisplatin can cause kidney dysfunction, although the damage is still very mild. Nguyen (8) in his literature review said that the creatinine serum level usually does not change until there is about $50 \%$ of kidney dysfunction. In acute kidney dysfunction, the glomerular filtration is reduced and therefore creatinine serum level is not accurate enough to illustrate the status of kidney function until the balance takes place, in which the process may need a relatively long period of time. Our study result is different from those in the study conducted by Hoek et al. (9) showing that the 3rd degree nephrotoxicity (creatinine serum level of $>3 x$ ) occurred more frequently in subjects who had received prior cisplatin chemotherapy compared to those who never received any chemotherapy ( $0 \%$ vs. $8 \%)$. The difference may be due to factors affecting creatinine serum level, which in our study were not controlled or measured. Some of those factors are age, sex, muscle mass, muscle metabolism and hydration status (8). Other researchers have also mentioned that hydration status, diuresis and electrolyte status of the patients may probably affect nephrotoxicity caused by cisplatinbased chemotherapy. Rades et al. (6) have reported that hydration and dieresis may protect the nephron of the group given cisplatin-based chemotherapy at the dose of $100 \mathrm{mg} / \mathrm{m} 2$ and Arunkumar et al. (7) have shown that cisplatin may induce nephrotoxicity through the mechanism of electrolyte reabsorption disorder in the kidney tubular cells.

Regarding the relatively new biomarkers for kidney dysfunction, our study showed that there was a significant delta difference of NGAL urinary levels between before and after treatment in three groups $(p=0.025)$. There was also a significant difference in a pair of groups, i.e. the group that had never received chemotherapy of $40 \mathrm{mg} / \mathrm{m} 2(\mathrm{p}=0.02)$ and in the group that had been treated with $75-100 \mathrm{mg} / \mathrm{m} 2$ chemotherapy $(p=0.02)$. Therefore, our study has demonstrated that measuring NGAL urinary level may detect kidney dysfunction. Some studies have also supported the results of our study. According to Shirali (10), the NGAL urinary or serum level will increase in those with acute kidney failure due to nephrotoxin such as cisplatin, aminoglycosides and other nephrotoxic drugs. Mishra et al. (11) have also demonstrated increased gene expression of NGAL in renal ischemia and the urinary level in mice can be rapidly increased within only 3 hours after cisplatin administration. It is also supported by Van Meer L et al. (4) who have suggested that the NGAL urinary level can be used as a biomarker for detecting early stage acute damage in cortical tubules of the kidney. The effect of accumulation and dose-dependent characteristic can be described by an obvious mean of difference in a pair of groups, i.e. those who had never received $40 \mathrm{mg} / \mathrm{m} 2$ chemotherapy and those who had received 75-100 $\mathrm{mg} / \mathrm{m} 2$ chemotherapy. Therefore, the measurement of NGAL urinary level still cannot replace the measurement of BUN serum level to detect kidney dysfunction in patients with advanced stage NPC who received cisplatin chemotherapy.

Furthermore, our study showed that the delta difference of KIM-1 urinary level in all three groups was not significantly different $(p=0.275)$. Therefore, the KIM1 urinary level still cannot be used as a biomarker for detecting kidney dysfunction due to cisplatin chemotherapy in patients with advanced stage NPC. 
Results of our study were in contrary to some experimental studies, which suggest that KIM-1 urinary level is a more sensitive biomarker than the conventional biomarkers of BUN and creatinine serum level in detecting kidney dysfunction caused by nephrotoxin in mice. Vaidya et al. (12) have measured KIM-1 urinary level in mice. Their study has shown that $\mathrm{KIM}-1$ is sensitive and the level is increased significantly in kidney injury. Tonumura et al. (11) have also shown that KIM-1 urinary level is increased significantly in mice within 24 hours following cisplatin administration, which also indicates that KIM-1 can detect the injury in kidney proximal tubule following cisplatin administration. However, data in human is still limited and KIM-1 reagent is not available for commercial purpose. Therefore, the validity of KIM-1 as biomarker for detecting kidney injury in human is still vague (13).

In general, our study has shown that NGAL and KIM-1 urinary levels have weak correlation with BUN and creatinine serum levels in almost all groups. It indicates that KIM-1 and NGAL urinary level still cannot be used to replace the role of BUN and creatinine serum levels in detecting mild kidney dysfunction caused by cisplatinbased chemotherapy in patients with advanced stage NPC. No previous study available that shows the correlation of BUN and creatinine serum levels with the KIM-1 and NGAL urinary levels. However, some researchers have different opinions about it.

Han WK et al. (14) suggest that measurement of KIM1 urinary level may serve as a diagnostic biomarker for acute kidney dysfunction. Their study has demonstrated increased KIM-1 urinary level can be detected within 12 hours after the development of kidney ischemia in patients with acute dysfunction caused by various other clinical etiologies. While Liangos et al. (15) showed that increased KIM-1 urinary level in 201 patients with acute kidney failure has significantly association with poor prognosis including increased mortality rate or increased needs in dialysis. Moreover, Chaturvedi et al. (13) in their study demonstrate that although KIM-1 examination may be used routinely in clinical studies, but its application in daily practice should be studied further and validated.

Furthermore, our study has also shown a strong correlation between creatinine serum level and NGAL urinary level, which was found only in a group, i.e. the group that never had chemotherapy of $75 \mathrm{mg}-100$ $\mathrm{mg} / \mathrm{m} 2$. The result is consistent with the result of a study conducted by Nguyen et al. (8), which has demonstrated that NGAL urinary level actually has a relatively good correlation with increased creatinine serum level. Therefore, NGAL may serve as a relatively sensitive biomarker for detecting early kidney injury.

There are some limitations in our study since the study was only performed at a single hospital with a small population size. Further studies with larger population and greater collaboration with other hospitals are necessary to provide greater impacts on daily clinical practice.

\section{CONCLUSION}

Our study shows that cisplatin can cause kidney dysfunction or has nephrotoxic characteristic. The measurement of NGAL urinary level can be used for detecting kidney dysfunction. However, it cannot replace the role of BUN serum level measurement to detect kidney dysfunction in patients with advanced stage NPC who received cisplatin chemotherapy. On the contrary, the measurement of KIM-1 urinary level still has not shown its role as a biomarker for detecting kidney dysfunction due to cisplatin chemotherapy in patients with advanced stage NPC.

Kidney dysfunction caused by cisplatin can accumulate in the kidney and the nephrotoxicity is in dose dependent manner. The effect of dose dependent shown by cisplatin in our study is apparent as the subject who had never received cisplatin and then got cisplatin at $40 \mathrm{mg} / \mathrm{m} 2$ had a lower delta difference BUN serum level than those who had been exposed by cisplatin at the dose of $75-100 \mathrm{mg} / \mathrm{m} 2$. It may occur because the subjects had less dose of cisplatin.

Further studies are necessary to evaluate the role of measuring KIM-1 and NGAL urinary levels as biomarker for kidney dysfunction in NPC patients who received cisplatin-based chemotherapy. The studies should be conducted with larger sample size and better method of study.

\section{DECLARATIONS}

The study was conducted in Cipto Mangunkusumo Hospital, Jakarta, Indonesia and data collection was performed after an ethical clearance had been issued by No.987/UN2.F1/ETIK/2016 from the Ethic Committee at Faculty of Medicine, University of Indonesia and No. LB.02.01/X2/10942016 from Cipto Mangunkusumo Hospital.

\section{REFERENCES}

1. Torre LA, Bray F, Siegel RL, Ferlay J, Lortet-Tieulent J, Jemal A. Global cancer statistics, 2012. CA Cancer J Clin. 2015 Mar;65(2):87-108.

2. Yao X, Panichpisal K, Kurtzman N, Nugent K. Cisplatin nephrotoxicity: a review. Am J Med Sci. 2007 Aug;334(2):115-24.

3. Tsang RY, Al-Fayea T, Au HJ. Cisplatin overdose: toxicities and management. Drug Saf. 2009;32(12):1109-22.

4. Meer LV, Moerland M, Cohen AF, Burggraaf J. Urinary kidney biomarkers for early detection of nephrotoxicity in clinical drug development. Br J Clin Pharmacol. 2014 Jun;77(6):947-57. 
5. Espeli V, Zucca E, Ghielmini M, Giannini O, Salatino A, Martucci $F$, et al. Weekly and 3-weekly cisplatin concurrent with intensity-modulated radiotherapy in locally advanced head and neck squamous cell cancer. Oral Oncol. 2012 Mar;48(3):266-71.

6. Rades D, Kronemann S, Meyners T, Bohlen G, Tribius S, Kazic N, et al. Comparison of four cisplatin-based radiochemotherapy regimens for nonmetastatic stage III/IV squamous cell carcinoma of the head and neck. Int J Radiat Oncol Biol Phys. 2011 Jul 15;80(4):1037-44.

7. Arunkumar $P$, Viswanatha $G$, Radheshyam N, Mukund $H$, Belliyappa $M$. Science behind cisplatin-induced nephrotoxicity in humans: A clinical study. Asian Pac J Trop Biomed. 2012 Aug;2(8):640-4.

8. Nguyen MT, Devarajan P. Biomarkers for the early detection of acute kidney injury. Pediatr Nephrol. 2008 Dec;23(12):2151-7.

9. Hoek J, Bloemendal KM, van der Velden LA, van Diessen JNA, van Werkhoven E, Klop W, et al. Nephrotoxicity as a dose-limiting factor in a high-dose cisplatin-based chemoradiotherapy regimen for head and neck carcinomas. Cancers. 2016;8(2):21.

10. Shirali AC, Pazhayattil GS. Drug-induced impairment of renal function. Int J Nephrol Renovasc Dis. 2014;7:45768.

11. Mishra J, Ma Q, Prada A, Mitsnefes M, Zahedi K, Yang J, et al. Identification of neutrophil gelatinase-associated lipocalin as a novel early urinary biomarker for ischemic renal injury. J Am Soc Nephrol. 2003 Oct;14(10):2534-43.

12. Vaidya VS, Ferguson MA, Bonventre JV. Biomarkers of acute kidney injury. Annu Rev Pharmacol Toxicol. 2008;48(1):463-93.

13. Chaturvedi S, Farmer T, Kapke GF. Assay validation for KIM-1: human urinary renal dysfunction biomarker. Int J Biol Sci. 2009;5(2):128-34.

14. Han WK, Bailly V, Abichandani R, Thadhani R, Bonventre JV. Kidney Injury Molecule-1 (KIM-1): a novel biomarker for human renal proximal tubule injury. Kidney Int. 2002 Jul;62(1):237-44.

15. Liangos $\mathrm{O}$, Perianayagam MC, Vaidya VS, Han WK, Wald $\mathrm{R}$, Tighiouart $\mathrm{H}$, et al. Urinary $\mathrm{N}$-acetyl-beta-(D)glucosaminidase activity and kidney injury molecule-1 level are associated with adverse outcomes in acute renal failure. J Am Soc Nephrol. 2007 Mar;18(3):904-12. 